\title{
Audit on effective handover of critically ill patients in intensive care units between shifts by the medical and nursing officers
}

\author{
$N$ Wanigasinghe ${ }^{I^{*}}$ \\ Consultant Anaesthetist ${ }^{l}$,General Hospital, Ampara, Sri Lanka.
}

Corresponding author: nandikawanigasinghe@yahoo.com

\begin{abstract}
Hand over is the process of transferring clinical data between health care professionals. Interruption to the continuity of care can take place during handover and result in poor patient outcome. The aim of this audit was to find out whether the handover practice between shifts in the Intensive care unit (ICU) is safe and effective. Data was collected by a self-administered questionnaire which was distributed among nursing and medical officers who were selected by convenience sampling of ICU's at the National Hospital of Sri Lanka (NHSL). It was identified that there are multiple deficiencies in the current hand over practice and need introduction of standardized hand over technique to our ICU's.
\end{abstract}

Keywords: hand over; critical care; safety; effectiveness; patient outcome

\section{Introduction}

Clinical handover refers to the transfer of professional responsibility and accountability of some or all aspects of care of a patient or group of patients to another person or professional group on a temporary or permanent basis. ${ }^{1}$ The aim of handover is to ensure health professionals involved in the care of patients have the same understanding and set of clinical priorities. ${ }^{2}$

Continuity of information is vital for the safety of critically ill patients. ${ }^{3}$ Ineffective handover has been found to result in poor patient outcome. Hand over is inevitable and essential due to shift working patterns in intensive care units. Clinical handover between shifts is a high risk activity for patient safety. ${ }^{4}$ Critical information of each patient should be transferred effectively and correctly in order to maintain continuity of care despite change in shifts among medical and nursing officers. Therefore, both medical and nursing staff has the responsibility of proper handover of their patients.

Several responsible bodies such as National Institute for Health and Care Excellence (NICE), National Patient Safety and quality health service standard shave described standards of hand over of patients in ICU.
Despite the existence of standard guidelines pertaining to hand-over, patient safety has been compromised due to non-adherence to these guidelines. Therefore, it is important to inquire into the current practice of hand over, in order to make necessary changes to improve and maintain standards of patient care in ICU.

\section{Methodology}

The aim of this audit is to determine whether the handover of ICU patients between shifts is occurring safely and effectively.

The objectives were

1. To assess the level of adherence to standard hand-over guidelines by the medical staff of various ICU's at NHSL

2. To assess the level of adherence to standard hand-over guidelines by the nursing staff of various ICU' sat NHSL

The study was conducted at NHSL which is a major tertiary care hospital in Sri Lanka. The hospital consists of 20 ICUs. Certain subspecialties have more than one ICU.

Eg - Cardiothoracic unit - 2 ICUs

Neuro-trauma unit - 6 ICUs

A patient turnover of about 40-50 takes place daily. To conduct the study five ICUs were selected by convenience sampling. 
1. Trauma ICU 6 beds

2. Surgical ICU 9 beds

3. Neurosurgical ICU1 8 beds

4. Neurosurgical ICU 28 beds

5. Medical ICU 9 beds

Currently about 500 nursing officers and 120 medical officers are employed in these ICUs. Two groups of ICU staff (one group of medical officers and one group of nursing officers) were selected from the above mentioned ICUs to conduct this audit. Four handovers take place per 24 hours for each group. That is night shift to morning shift, morning shift to afternoon shift, afternoon shift to night shift and night shift to following day morning shift. Though, both groups do four handovers per 24 hours the shift change time is different in the two groups.

22 medical officers and 37 nursing officers from five ICUs participated in this study. A 13 item selfadministered questionnaire was used to collect data. The questionnaire consisted of three main topics. Data was collected prospectively during shift changeovers over a period of 24 hours.

\section{Results}

The total number of participants was 59. The data was analyzed separately for nursing and medical officers.

General information

$\underline{\text { Medical officers }}$

Figure 1: Distribution of medical officers according to their grade

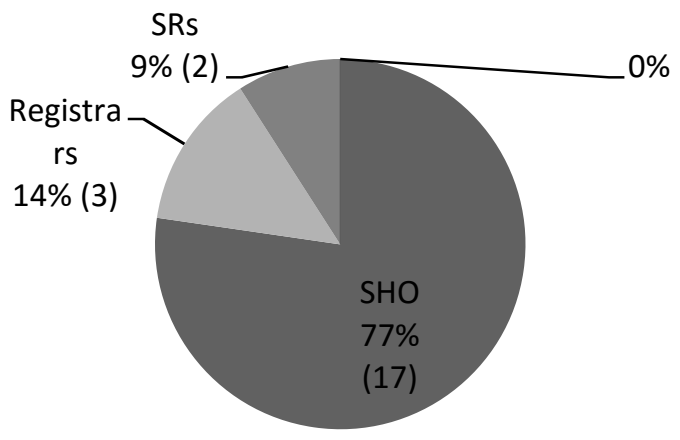

Table 1: Distribution of medical officers according to their experience in ICU

\begin{tabular}{|l|l|l|}
\hline $\begin{array}{l}\text { Experience in ICU } \\
\text { (years) }\end{array}$ & Number & $\begin{array}{l}\text { Percentage } \\
(\%)\end{array}$ \\
\hline$<1$ & 5 & 22.73 \\
\hline $1-5$ & 10 & 45.45 \\
\hline $6-10$ & 4 & 18.19 \\
\hline $11-15$ & 3 & 13.63 \\
\hline Total & 22 & 100.00 \\
\hline
\end{tabular}

Table 2: Distribution of Medical officers according to ICU

\begin{tabular}{|l|l|l|}
\hline specialty & $\begin{array}{l}\text { Number } \\
\text { of medical } \\
\text { officers }\end{array}$ & $\begin{array}{l}\text { Percentage } \\
(\%)\end{array}$ \\
\hline medical & 4 & 18.19 \\
\hline surgical & 8 & 36.37 \\
\hline NSU & 5 & 22.72 \\
\hline Trauma & 5 & 22.72 \\
\hline Total & 22 & 100.00 \\
\hline
\end{tabular}

\section{Nursing Officers}

Figure 2: Distribution - according to their grade

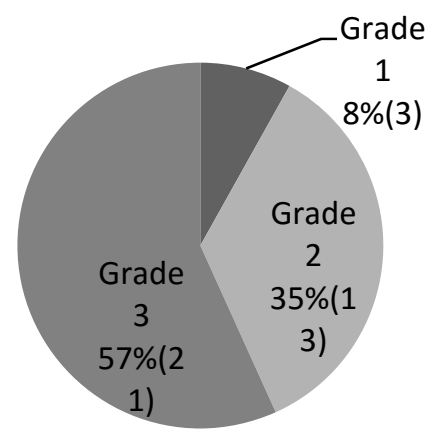

Table 3: Distribution of Nursing Officers according to the experience in ICU

\begin{tabular}{|l|l|l|}
\hline $\begin{array}{l}\text { Experience } \\
\text { (years) }\end{array}$ & Number & $\begin{array}{l}\text { Percentage } \\
(\%)\end{array}$ \\
\hline$<1$ & 3 & 8.10 \\
\hline $1-5$ & 23 & 62.17 \\
\hline $6-10$ & 8 & 21.62 \\
\hline $11-15$ & 2 & 5.40 \\
\hline$>15$ & 1 & 2.71 \\
\hline Total & 37 & 100.00 \\
\hline
\end{tabular}


Table 4: Distribution of Nursing Officers according to ICU

\begin{tabular}{|l|l|l|}
\hline $\begin{array}{l}\text { Specialty } \\
\text { of ICU }\end{array}$ & $\begin{array}{l}\text { Number of } \\
\text { nursing } \\
\text { Officers }\end{array}$ & $\begin{array}{l}\text { Percentage } \\
(\%)\end{array}$ \\
\hline Medical & 8 & 21.64 \\
\hline Surgical & 10 & 27.02 \\
\hline NSU & 9 & 24.32 \\
\hline Trauma & 10 & 27.02 \\
\hline Total & 37 & 100.00 \\
\hline
\end{tabular}

\section{Place and method of handover}

\section{Medical Officers}

All (100\%) of the medical handovers took place at bedside. More than two thirds $68.18 \%$ (15/22) handovers were one incoming to one outgoing medical officer. All (100\%) medical officers did face to face verbal handover while $13.63 \%(3 / 22)$ did both verbal and documented handovers. In $22.72 \%(5 / 22)$ occasions only one patient was handed over by outgoing medical officer to incoming medical officer. $>5$ patients were handed over by outgoing medical officer to incoming medical officer in $36.36 \%(8 / 22)$ handovers. The averrage duration per handoveris 36.81 minutes and it rangedbetween $10-90$ minutes.

\section{Nursing Officers}

Majority $91.89 \%$ (34/37) of handovers took place at bedside and $8.10 \%(3 / 37)$ handovers took place at ICU nurses' office room. 81.09\% (30/37) of handovers involved only two nursing officers. That was one incoming nursing officer to one outgoing nursing officer. Similar to medical officers $100 \%$ of the nursing staff did verbal face to face hand over. In addition $89.18 \%$ (33/37) of them were documented handovers. More than ninety percent $(94.59 \%$ - 35/37) of nursing handovers were one patient handovers. 2 patients and 5 patients were handed over in similar number of handovers ( 2 handovers each $-2.7 \%$ ) done by nursing officers
Distractions during handover

\section{Medical Officers}

Figure 3: Presence of distractions among Medical Officers

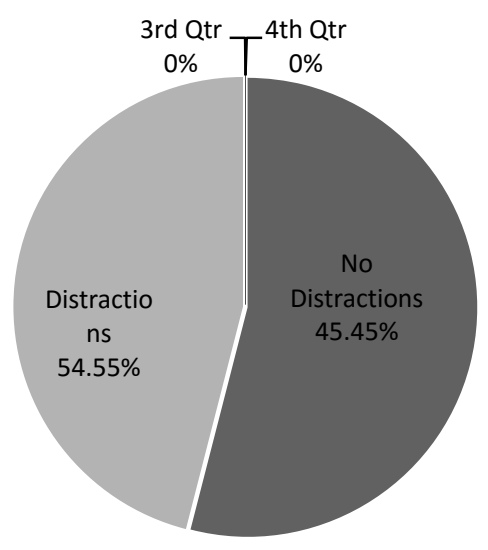

Figure 4: Modes of distraction

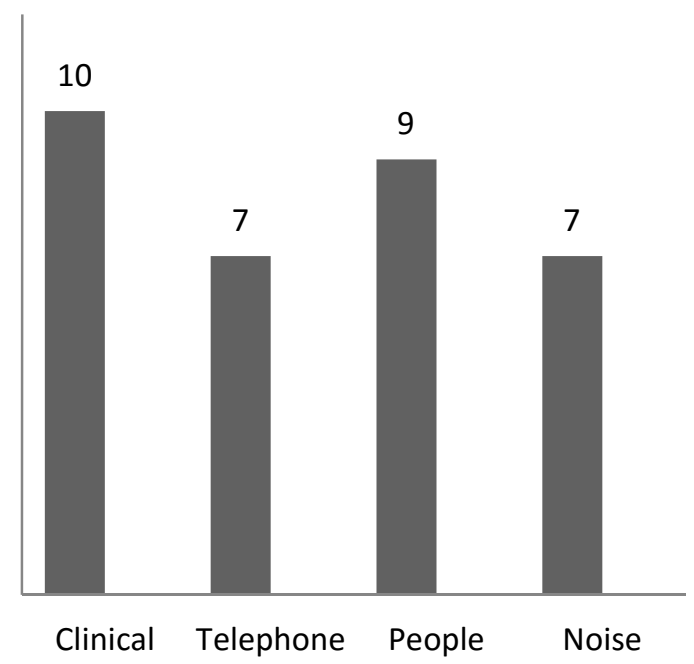


Figure 5: Clinical distractions

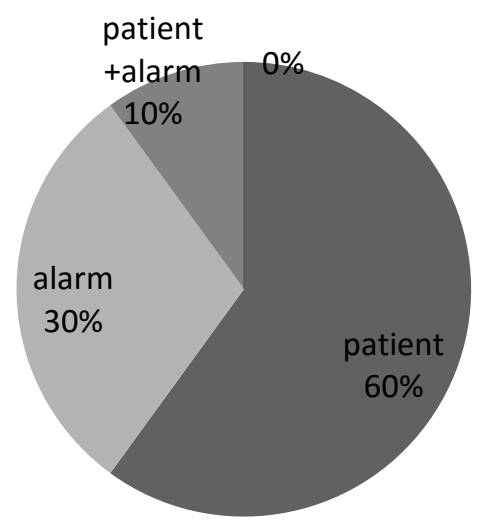

Patient factors which distracted the medical officers during the handover process were complaints of pain, difficulty in breathing, low urine output, etc.

Figure 6: Distractions for medical officers according to the number of distractions

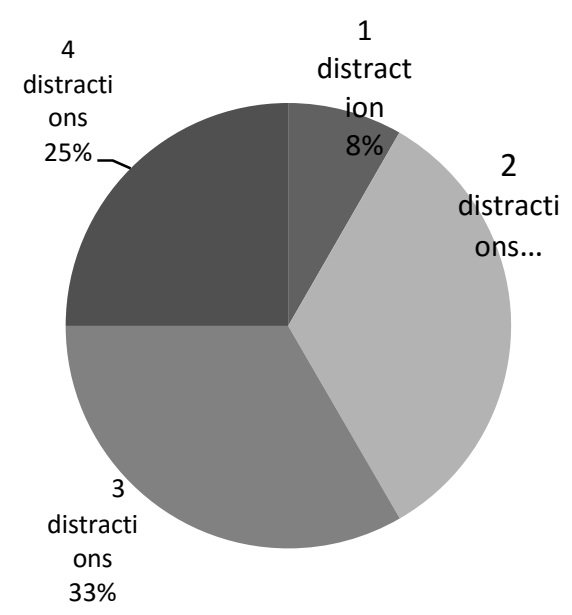

$33 \%$ and $34 \%$ of handovers by medical officers were distracted by 3 and 4 distractions respectively. $8 \%$ of medical handovers were distracted once only.

\section{Urgency of distractions}

Medical officers got disturbed equally from urgent and non-urgent distractions. (The distractions which needed immediate attention were considered as urgent).

\section{Nursing Officers}

Figure 7: Distractions during hand over

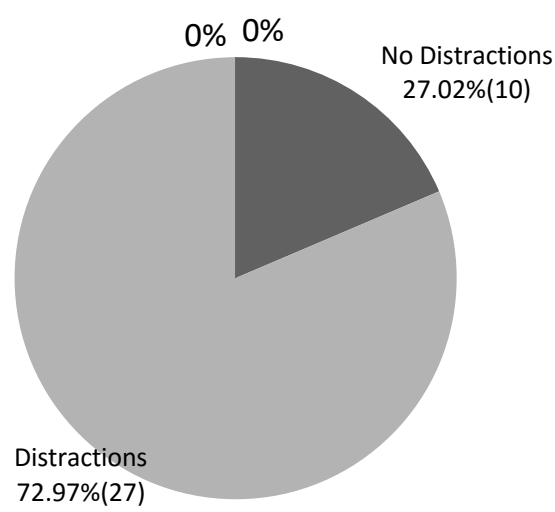

\section{$\underline{\text { Modes of Distractions }}$}

Figure 8: Modes of distractions among nursing staff

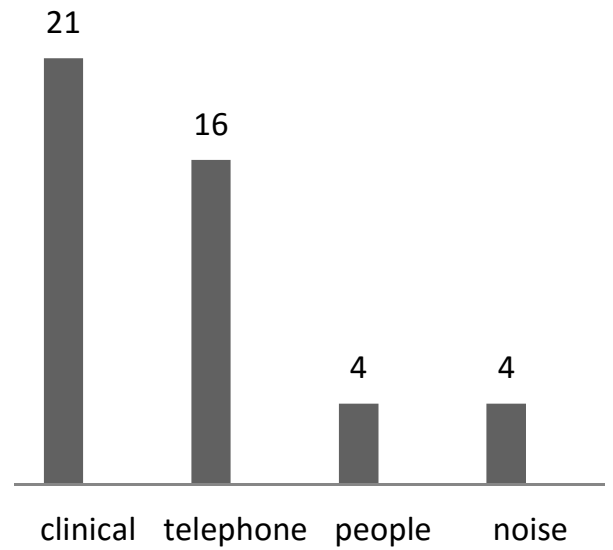


Figure 9: Nursing staff - Number of distractions during handover

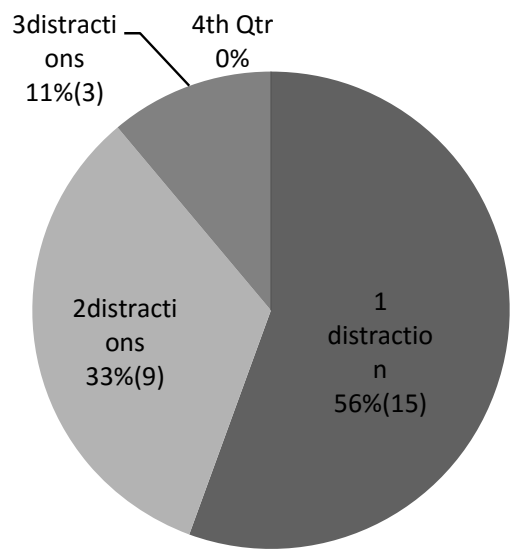

Urgency of distraction

More than half $(59.25 \%)$ of distractions were urgent and $40.74 \%$ were non urgent distractions.

Educational input for hand over

\section{Medical staff}

Medical officers had learnt about hand over from senior medical officers $(68.18 \%)$, through experience $(63.63 \%)$ and training programs (18.18\%). 27.27\% (6/22) medical officers had handover protocols in their ICUs and $72.72 \%$ $(16 / 22)$ did not have protocols.

$9.09 \%(2 / 22)$ mentioned that guidelines/protocols are not important while $90.09 \%$ (20/22) thought that they are important for patient safety.

\section{Nursing staff}

Over one third $(35.13 \%$ - 13/37) of nursing officers had their own handover protocols in their ICUs and $64.80 \%$ (24/37) did not have such guidelines or protocols. All nursing officers think that ICUs protocols are important.

\section{Discussion}

Effective handover practice is crucial to ensure safety of the critically ill patients between shift changes. Deficiencies in handover practice can compromise the patients' health due to lack of continuity of patient care. ${ }^{2}$

In this study $100 \%$ of medical officer handovers and $91.89 \%$ of nursing handovers had taken place at the bedside. The bedside handover is beneficial as it allows the incoming staff to see and if possible to talk to the patient, observe and monitor at the same time of handover and avoid unattended critically ill patient during handover. ${ }^{4}$ The handover should occur in a designated area and at a designated time without avoidable interruption. ${ }^{3}$ In this study none of the handovers took place in a designated area. Some nursing officers had utilized their office room for hand over.

The staff involved in the handover plays an important role in this process. The ideal model includes all grades of staff from each specialty, subspecialty or ward as appropriate. ${ }^{6}$ There should be clear identification of who is leading the handover. ${ }^{5}$

In this study $81.08 \%$ of nursing handovers involved 2 persons and that is from outgoing to incoming nursing officer.

The handover should occur at a fixed time and be of sufficient length and this period should be known to all staff who is involved in handover. ${ }^{5,6}$ The duration of handover in this study ranged from 15-45 minutes for nursing staff and 1590minutesfor medical staff.

Both groups had a $100 \%$ verbal face to face handover and in addition to that $86.48 \%$ (32/37) of nursing staff had done documented handover. Face to face handover is more efficient and is an effective mode of transferring information and it gives the opportunity to clarify information during handover. Written handover minimizes the risk of forgetting tasks as it is been documented. ${ }^{4} \mathrm{An}$ electronic handover system that works effectively contribute to safety of the patient even though, this has not been demonstrated by research. ${ }^{5}$

The proposed standard or target for best practice is $<10 \%$ of handovers being interruptedfor $100 \%$ of hand over processes. ${ }^{3}$ That allows for emergencies such as a cardiac arrest. Use of designated areas for handover minimizes disturbances. ${ }^{6}$ In this study $72.97 \%$ of nursing handovers were interrupted and $59.25 \%$ were non urgent. $54.55 \%$ medical handovers were interrupted and the percentages of urgent and non-urgent distractions were equal. The majority of distractions were due to clinical reasons. Telephone calls also affected the 
handover process significantly (43.24\% for nursing staff and $58.33 \%$ for medical staff).

It is important to educate ICU staff with regard to hand over practices for better patient care and outcome. Only $21.62 \%$ nursing staff and $18.18 \%$ medical staff had attended training programs on handover. $64.86 \%$ and $83.78 \%$ of nursing staff had learnt about ICU handover by experience and senior advice respectively. The respective values for medical officers are $63.63 \%$ and $68.18 \%$.

There should be an effective structure for communication at handover and information should be exchanged to pass on tasks. $27.27 \%$ $(6 / 22)$ medical officers and $35.13 \%$ nursing staff had protocols on handover in their ICUs.

$100 \%$ of nursing staff and $90.09 \%$ of medical officers think that it is important to have protocols or guidelines on handover practice in their ICUs.

Good hand over practice is beneficial to the staff educationally, professionally and psychologically (reduction of stress and job satisfaction). ${ }^{5}$

\section{Conclusion}

It is essential to ensure that critical information is effectively communicated as an essential component of risk management and patient safety as continuity of information is vital to the safety of the patients. The number of individuals potentially caring for a patient during their hospital stay has increased and the need for comprehensive handover of clinical information has become more important than ever. This study identified that there are multiple deficiencies in current handover practices in the ICUs with regard to structure, documentation, location, training, etc. Improvements in clinical handover can be achieved by introducing structured and standardized handover technique and conducting practice-based handover training to the relevant staff. After above implementations a re-audit is recommended to assess the changes of the current practice.

\section{Acknowledgement}

Dr Enoka Wickramasinghe, Dr Jayantha Jayasuriya, Dr J Willers, Dr Marie Fernando, Professor Anuja Abayadeera and all the staff who participated in the study

\section{References}

1. Australian Commission on safety and quality in health, National safety and quality health service standards, Standard 6 - Clinical handover, September 2012, page 44-47

2. ClinicalAudits.com ISSN 2042-4779

3. Rivers J, Peden C. Quality and safety of handover in intensive care - 10.3, Intensive care medicine, Section 10, Dr Morgan G, Royal College of Anaesthetists - Raising the Standard : a compendium of audit recipes , 3rd Edition, 2012, Page 262-263

4. Pascoe H, Gill SD, Hughes A, McCall-White M. Clinical handover: An audit from Australia. Australian medical journal 2014;7(9):363-371 http://dx.doi.org/10.4066/AMJ.2014.2060 PMid:25324901 PMCid:PMC4185328

5. Safe handover: safe patients, Guidance on clinical handover for clinicians and managers The Newcastle upon Tyne Hospitals NHS Foundation trust, Clinical handover policy, October 2010 